\title{
Structural and Dielectric Studies of Gd Doped ZnO Nanocrystals at Room Temperature
}

\author{
P. U. Aparna, N. K. Divya, P. P. Pradyumnan* \\ Department of Physics, University of Calicut, Malappuram, India \\ Email: "drpradyumnan@gmail.com
}

Received 8 January 2016; accepted 12 February 2016; published 17 February 2016

Copyright (C) 2016 by authors and Scientific Research Publishing Inc.

This work is licensed under the Creative Commons Attribution International License (CC BY). http://creativecommons.org/licenses/by/4.0/

(c) (i) Open Access

\begin{abstract}
Gadolinium doped Zinc oxide $\left(\mathrm{Zn}_{1-x} \mathrm{Gd}_{x} 0\right)$ nanocrystals with different percentage of Gd content $(x$ $=0,0.2,0.4,0.6,0.8$ ) have been prepared by the solid state reaction method. The structural, morphological and chemical studies of the samples were performed by X-ray diffraction (XRD), Scanning electron microscope (SEM) and Energy dispersive X-ray (EDX) analysis. The XRD spectra confirm that all the samples have hexagonal wurtzite structure. Decrease in average crystallite size with an increase in Gd concentration is observed in XRD. SEM images show that the grain size of undoped $\mathrm{ZnO}$ is larger than the Gd doped $\mathrm{ZnO}$, specifying the hindrance of grain growth upon Gd doping. The chemical composition of the samples was confirmed using Energy dispersive $\mathrm{X}$-ray (EDX) analysis. The variation of dielectric constant $\left(\varepsilon_{r}\right)$, dielectric loss $(\tan \delta)$ and AC conductivity as a function of frequency is studied at room temperature in a frequency which ranges from 100 $\mathrm{Hz}$ - 4.5 MHz by using LCR Hi TESTER. All the samples exhibit the normal dielectric behavior, i.e. decreases with increase in frequency which has been explained in the light of Maxwell-Wagner model. The dielectric constant and dielectric loss can be varied intensely by tuning Gd concentration in $\mathrm{Zn}_{1-x} \mathrm{Gd}_{x} \mathrm{O}$ compounds.
\end{abstract}

\section{Keywords}

XRD, SEM, EDX, Dielectric Constant, AC Conductivity, Maxwell-Wagner Model

\section{Introduction}

$\mathrm{ZnO}$ is a versatile semiconductor having a wide band gap of $3.37 \mathrm{eV}$ and large exciton energy of 60 meV which

${ }^{*}$ Corresponding author.

How to cite this paper: Aparna, P.U., Divya, N.K. and Pradyumnan, P.P. (2016) Structural and Dielectric Studies of Gd Doped ZnO Nanocrystals at Room Temperature. Journal of Materials Science and Chemical Engineering, 4, 79-88.

http://dx.doi.org/10.4236/msce.2016.42009 
crystallizes in hexagonal wurtzite structure. Due to its unique physical and chemical properties, it has a wide spread application in solar cells, gas sensors, UV light emitters and surface acoustic wave (SAW) devices [1]-[3]. The lack of centre of symmetry in the wurtzite structure of $\mathrm{ZnO}$ crystals give rise to its piezoelectric and pyroelectric properties [2]. Therefore $\mathrm{ZnO}$ can be used for piezoelectric and pyroelectric applications such as transducer, actuator, IR sensors and energy generator. Some of the promising features of $\mathrm{ZnO}$ include its radiation hardness, biocompatibility and its high transparency in the visible region.

Doping $\mathrm{ZnO}$ with rare earth ions is of great interest for optoelectronics and spintronic applications [4]. The peculiar properties exhibited by rare earth ions are due to its intra f-shell transition. A good number of reports in the literature propose that doping with rare earth elements causes an enhancement in optical and magnetic properties of ZnO. K. Jayanthi et al. [5] observed a sharp and intense visible line emission from $\mathrm{Nd}^{3+}$ doped $\mathrm{ZnO}$ nanopowders. Achamma et al. [6] studied the luminescence properties in Ce doped $\mathrm{ZnO}$ nanocrystals. John and Rajakumari [7] reported the presence of ferromagnetic properties in Er doped ZnO nanocrystals. Reports on the dielectric and ferroelectric studies of rare earth ion doped $\mathrm{ZnO}$ are found limited. Nidhis Sinha et al. [8] reported about the dielectric and ferroelectric properties of Ce doped ZnO nanorods. Recently, Divya et al. [9] [10] studied the optical and dielectric properties of Er doped ZnO. The study of dielectric properties is concerned with the storage and dissipation of electric and magnetic energy in materials which helps to improve the design and quality of the devices. Many workers have studied the structural, optical and magnetic properties of Gd doped $\mathrm{ZnO}$ [11]-[16]. But dielectric studies on $\mathrm{Gd}$ doped $\mathrm{ZnO}$ are not found elsewhere. The aim of the present work is to synthesize Gd doped $\mathrm{ZnO}$ via the solid state reaction route and to examine whether the dielectric constant of these materials can be enhanced compared to pure $\mathrm{ZnO}$. In this paper, we have investigated the effect of Gd doping in $\mathrm{ZnO}$ on its structural, morphological and dielectric properties.

\section{Experimental Details}

Gd doped $\mathrm{ZnO}$ were synthesized by solid state reaction route to study their structural and dielectric properties. The chemicals used in the experiment are $\mathrm{ZnO}$ (99.99\% pure), $\mathrm{Gd}_{2} \mathrm{O}_{3}\left(99.99 \%\right.$ pure) and $\mathrm{LiOH} \cdot \mathrm{H}_{2} \mathrm{O}(99.99 \%$ pure). These chemicals were weighed using an electronic balance in accordance with the required stoichiometry. These materials were homogeneously mixed using an agate mortar for sufficient time to get fine powders. $\mathrm{LiOH}$ is an inorganic and water soluble compound used as a heat transfer medium for the synthesis of Gd doped ZnO. The prepared samples were mixed with ethanol and made into slurry. It is then dried in an oven for 1 hour at $100^{\circ} \mathrm{C}$. After drying, the mixture was ground for 1 hour and made into pellets using hydraulic pelletizer. These pellets were sintered at $900^{\circ} \mathrm{C}$ for 4 hours in a high temperature furnace. The pellets were again ground and used as samples for the studies.

The XRD patterns of powder samples were attained by Rigaku Miniflex 600 X-ray diffractometer. Surface morphology and chemical composition of the samples were respectively examined by scanning electron microscopy (SEM) and energy dispersive X-ray analysis (EDX). For dielectric and AC conductivity measurements, the powder samples were made into pellets using hydraulic pelletizer of thickness 1 - $2 \mathrm{~mm}$ and of diameter 10 $\mathrm{mm}$ by applying pressure of $130 \mathrm{Kg}-\mathrm{cm}^{2}$ for 1 minute. The dielectric constant, dielectric loss and AC conductivity were measured using HIOKI 3532-50 LCR Hi TESTER for a frequency range from $100 \mathrm{~Hz}$ to $4.5 \mathrm{MHz}$.

\section{Result and Discussion}

\subsection{XRD Analysis}

$\mathrm{X}$-ray diffraction pattern of undoped and gadolinium doped $\mathrm{ZnO}, \mathrm{Zn}_{1-x} \mathrm{Gd}_{x} \mathrm{O}$ whereas $(x=0, x=0.2, x=0.4, x=$ $0.6, x=0.8$ ) are shown in Figure 1. These patterns have been compared with standard JCPDS data. All the XRD peaks can be indexed to a wurtzite structure of $\mathrm{ZnO}$. It is found that up to $0.6 \mathrm{wt} \%$ of $\mathrm{Gd}$ doping, there were no extra peaks which implies that $\mathrm{Gd}^{3+}$ ion perfectly replaced $\mathrm{Zn}^{2+}$ ion in the crystal matrix. Further increase of $\mathrm{Gd}^{3+}(x \geq 0.8)$, extra peaks arises and is shown in Figure 2.

It is noticeable that the XRD peaks of doped samples were found shifted towards the higher $2 \theta$ values. The peak shift observed in the XRD pattern for the peak corresponding to the plane (101) depicted in Figure 3 . This shift is mainly due to the incorporation of larger sized $\mathrm{Gd}^{3+}(r=0.94 \AA)$ than $\mathrm{Zn}^{2+}(r=0.74 \AA)$ in the hexagonal wurtzite structure. This indicates that the doped Gd atoms substitute $\mathrm{Zn}$ atoms and the crystal structure remains unchanged. 
Variation of lattice parameters with Gd concentration is shown in Table 1. Crystallite sizes were also estimated from XRD pattern using the Scherrer formula.

$$
t=K \lambda / \beta \cos \theta,
$$

where $t$ is the crystallite size, $K$ is the shape factor, $\lambda$ is the wavelength of the incident X-ray radiation, $\theta$ is the bragg angle and $\beta$ is the full width at half maximum (FWHM) in radian of the peak with given (hkl) value.

It was found that crystallite size decreases with increasing Gd concentration (up to 0.6 wt.\% of Gd) as shown in Table 2. This is due to the distortion of host $\mathrm{ZnO}$ lattice by $\mathrm{Gd}^{3+}$ ions, which actually reduces the nucleation and subsequent growth rate of $\mathrm{ZnO}$ crystal. Similar results were reported by D. Mithal et al. [17], a decrease of crystallite size with increase in concentration of $\mathrm{Gd}$ in $\mathrm{Zn}_{1-x} \mathrm{Gd}_{x} \mathrm{O}$.

It could be noted that the crystallite size of $0.8 \mathrm{wt} . \% \mathrm{Gd}$ doped $\mathrm{ZnO}$ is larger when compared to other concentrations and it tending to the size of pure $\mathrm{ZnO}$ crystallites on higher doping percentage. This shows that the solu-

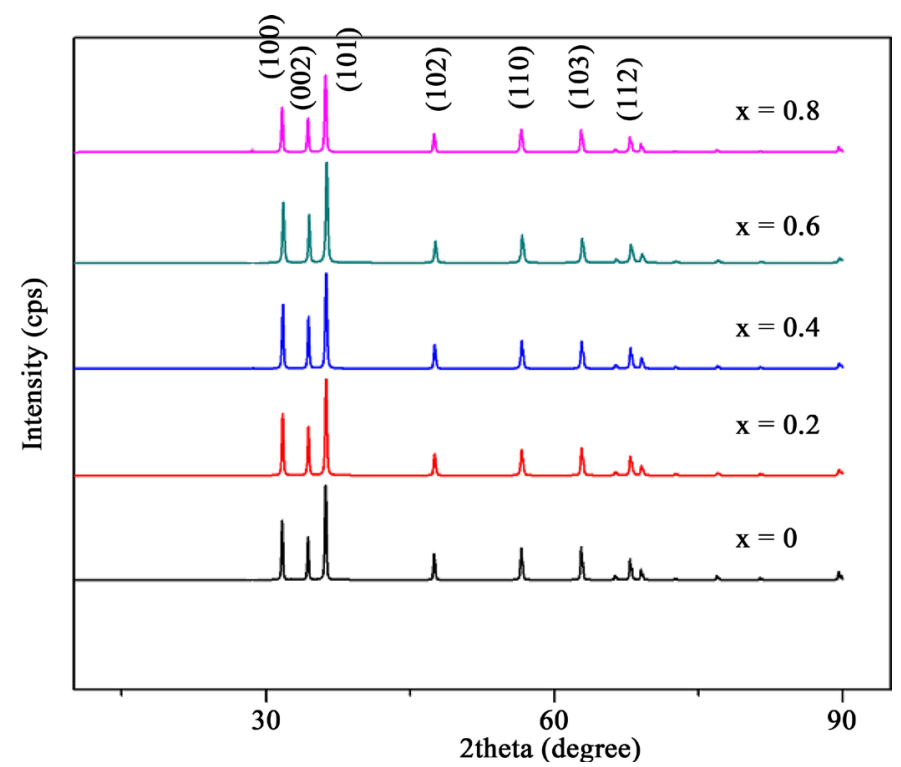

Figure 1. XRD pattern for Gd doped ZnO.

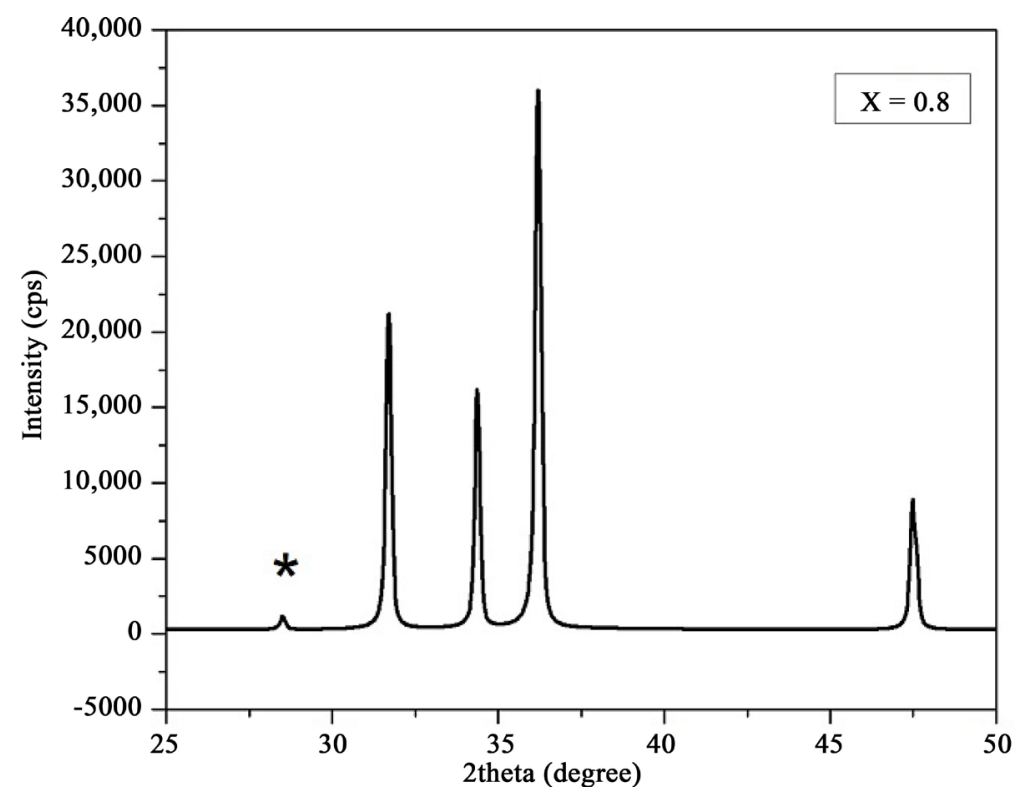

Figure 2. XRD pattern of 0.8 wt.\% Gd doped ZnO. 


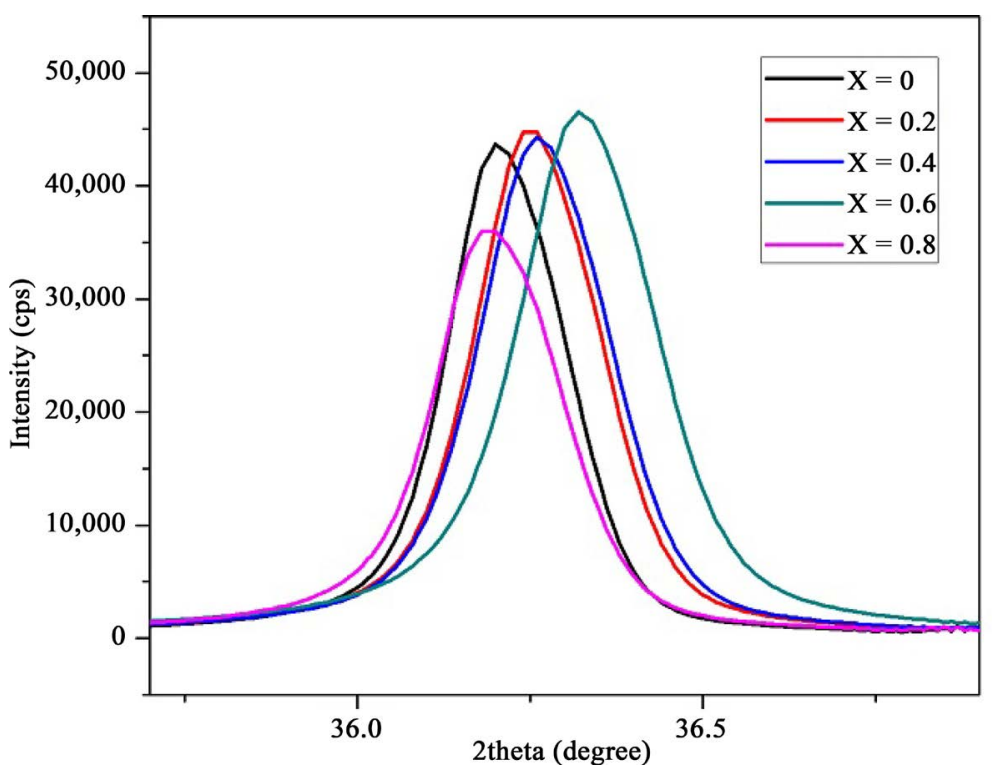

Figure 3. Peak shift observed in the XRD pattern for the peak corresponding to (101) plane.

Table 1. Variation of lattice parameters with Gd concentration.

\begin{tabular}{cccc}
\hline Concentration of gadolinium (wt.\%) & $\mathrm{a}(\AA)$ & $\mathrm{c}(\AA)$ & $\mathrm{c} / \mathrm{a}$ \\
0 & 3.25331 & 5.21137 & 1.6018 \\
0.2 & 3.25118 & 5.20893 & 1.6021 \\
0.4 & 3.25012 & 5.20787 & 1.6023 \\
0.8 & 3.24732 & 5.20111 & 1.6016 \\
\hline
\end{tabular}

Table 2. Variation of average crystalline size, volume and specific surface area with Gd concentration.

\begin{tabular}{cccc}
\hline Concentration of gadolinium (wt.\%) & Average crystalline size $(\mathrm{nm})$ & Cell volume $\left(\AA^{3}\right)$ & Specific surface area $\left(\mathrm{m}^{2} / \mathrm{g}\right) \times 10^{3}$ \\
\hline 0 & 64.5 & 47.79 & 16.435 \\
0.2 & 52.1 & 47.71 & 20.346 \\
0.4 & 51.2 & 47.67 & 20.704 \\
0.6 & 47.1 & 47.52 & 22.506 \\
0.8 & 56.0 & 47.83 & 18.929 \\
\hline
\end{tabular}

bility limit of $\mathrm{Gd}$ ion in the $\mathrm{ZnO}$ crystal lattice is close to $0.6 \mathrm{wt} \%$ and excess Gd ions may precipitate out on the particle surface.

The volume of the unit cell for hexagonal system has been calculated using the formula, $V=0.866 a^{2} c$. The unit cell volume is completely dependent on lattice constants. From the Table 2, it is clear that the volume of the unit cell decreases with small concentration of gadolinium. This is because of the perfect replacement of $\mathrm{Zn}^{2+}$ by $\mathrm{Gd}^{3+}$ in the crystal lattice. But as the concentration of doping increases, the gadolinium ion will also incorporate at the interstitial position, as a result lattice parameter changed and thus unit cell volume found increased.

The specific surface area of the crystallites of the samples was also determined using XRD. The specific surface area is a material property of solids which measures the total surface area of the crystallites present in per unit of mass. It is an important parameter that can be used to determine the type and properties of a material. It is particularly significant for adsorption, heterogeneous catalysis, and reactions on surfaces. The specific surface area can be calculated by Sauter formula,

$$
S=6 \times 10^{3} / D_{p} \cdot \rho
$$


where $S$ is the specific surface area, $D_{p}$ is the size of the particle and $\rho$ is the density of $\mathrm{ZnO}$ which equals to $5.606 \mathrm{~g} / \mathrm{cm}^{3}$. Table 2 shows that, the specific surface area (S) of gadolinium doped $\mathrm{ZnO}$ nanocrystals was found to increase from $9.511 \mathrm{~m}^{2} \cdot \mathrm{g}^{-1}$ to $13.025 \mathrm{~m}^{2} \cdot \mathrm{g}^{-1}$ as the concentration of gadolinium in $\mathrm{ZnO}$ increased up to 0.6 wt.\%. At 0.8 wt.\% it becomes decreased due to the presence of gadolinium oxide in the sample.

\subsection{Scanning Electron Microscopy}

The SEM technique was employed to find the size and distribution of particles in the materials. Figure 4 displays the surface morphology of undoped and Gd doped $\mathrm{ZnO}$ nanocrystalline powders. It can be seen that the samples prepared have smaller particle sizes.

Microstructural variation of $\mathrm{Gd}$ doped $\mathrm{ZnO}$ compared to pure $\mathrm{ZnO}$ is due to the significant difference in the ionic radius of $\mathrm{Gd}^{3+}$ related to the $\mathrm{Zn}^{2+}$ in $\mathrm{ZnO}$. Ionic radius of $\mathrm{Gd}^{3+}$ is $0.938 \AA$, which is higher to $\mathrm{Zn}^{2+}(0.74 \AA$ ) . Therefore higher radius $\mathrm{Gd}^{3+}$ may suppress the formation of larger nuclei during the crystallization process in $\mathrm{ZnO}$. As a result of this, there is a reduction in the grain size happens. Thus Gd incorporation leads to a reduction

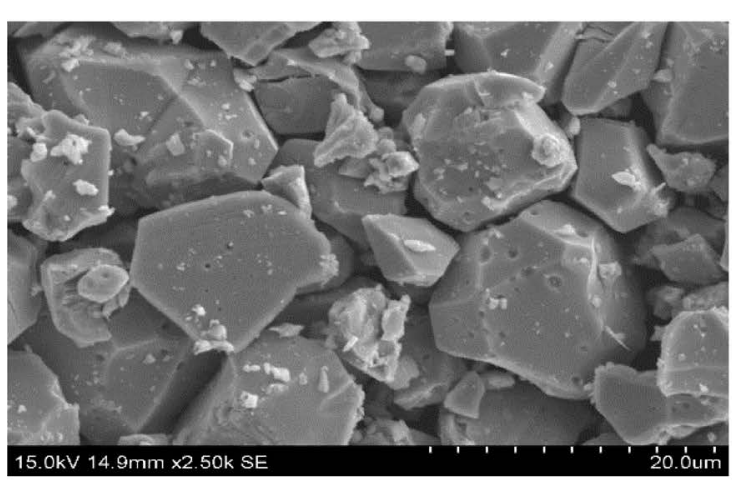

(a)

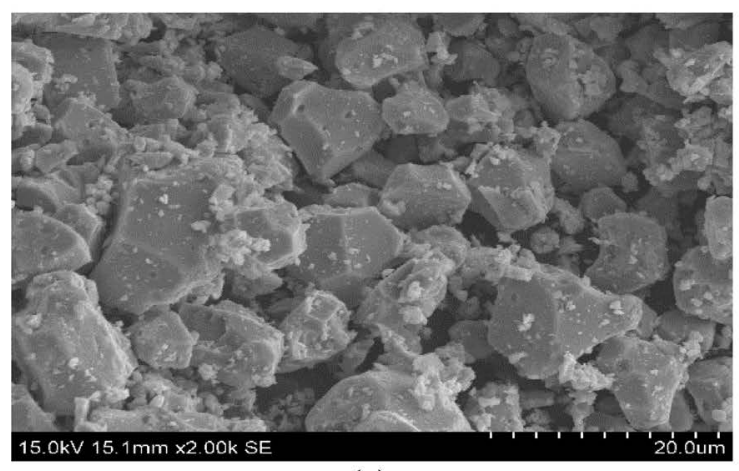

(c)

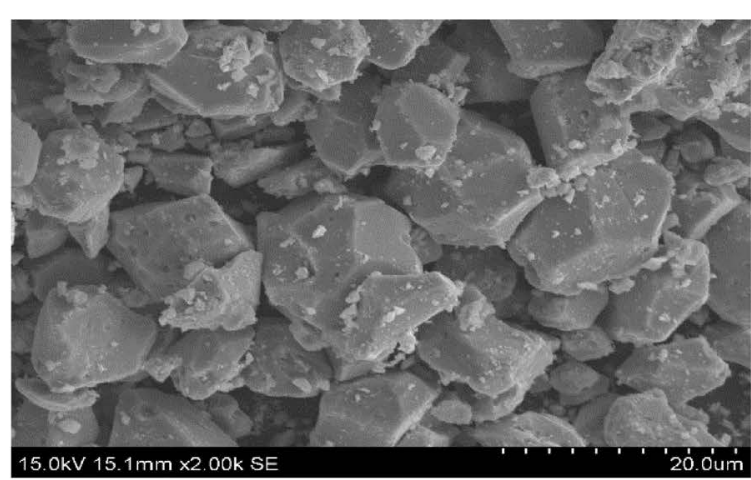

(b)

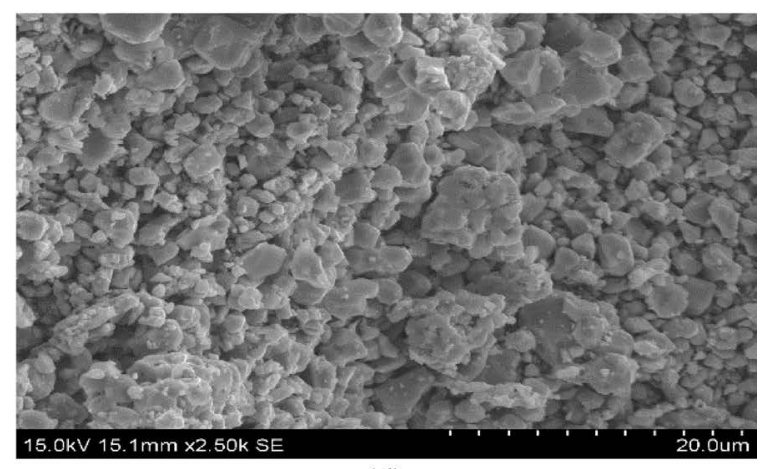

(d)

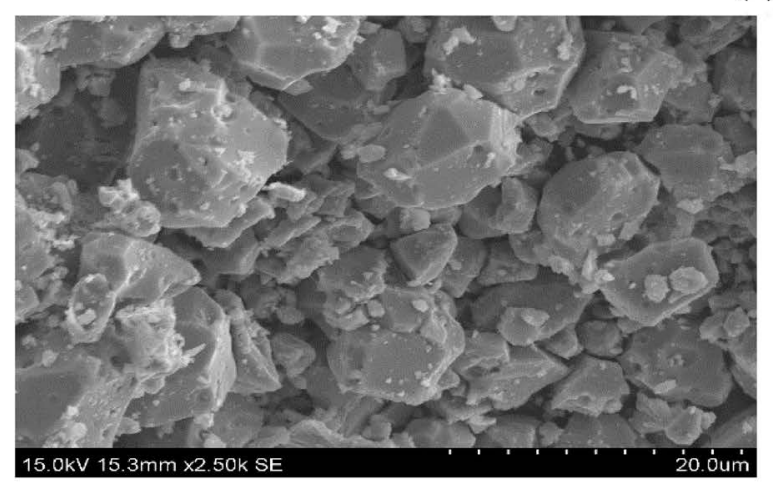

(e)

Figure 4. SEM images of (a) Pure $\mathrm{ZnO}$ (b) $\mathrm{Zn}_{0.8} \mathrm{Gd}_{0.2} \mathrm{O}$ (c) $\mathrm{Zn}_{0.6} \mathrm{Gd}_{0.4} \mathrm{O}$ (d) $\mathrm{Zn}_{0.4} \mathrm{Gd}_{0.6} \mathrm{O}$ (e) $\mathrm{Zn}_{0.2} \mathrm{Gd}_{0.8} \mathrm{O}$. 


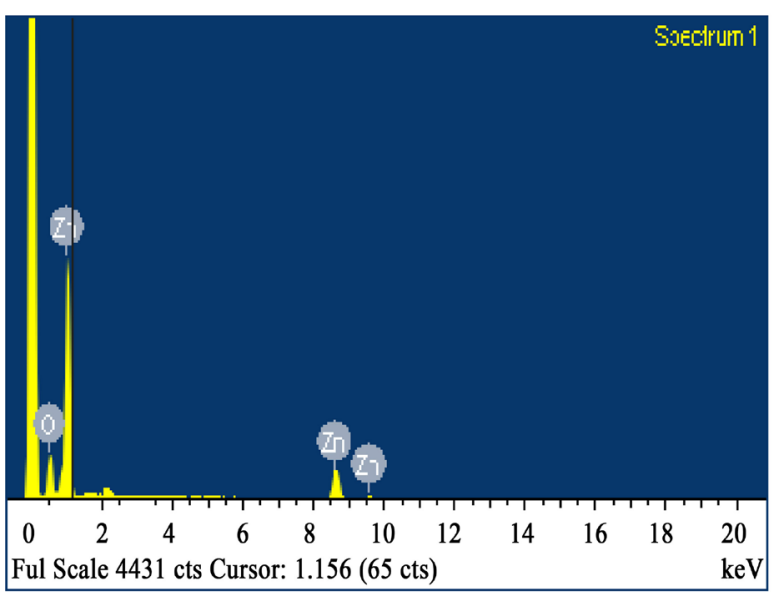

(a)

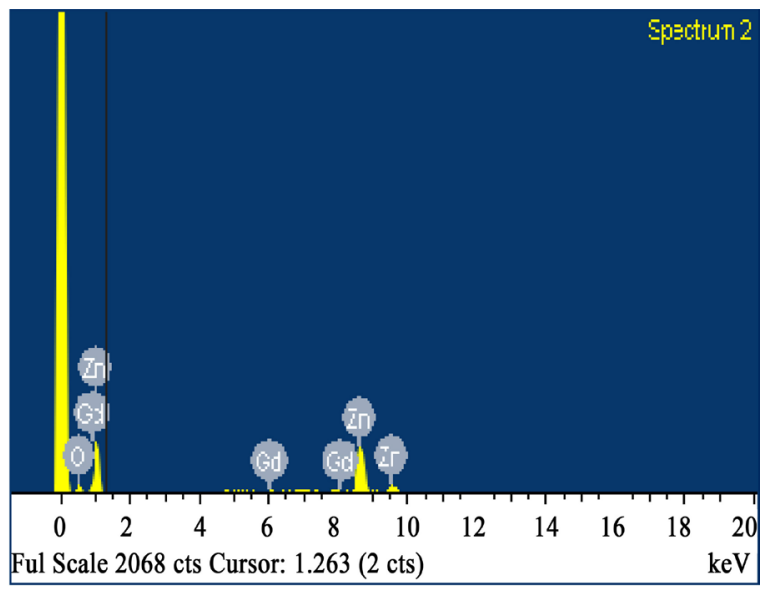

(b)

Figure 5. EDX spectrum of (a) $\mathrm{ZnO}$ (b) $\mathrm{Zn}_{0.2} \mathrm{Gd}_{0.8} \mathrm{O}$.

in grain or crystallite size.

Another reason for the reduction of grain size is due to physiochemical effect. During the compound formation and sintering time, the larger radius Gd ion will enter into the lattice partially and the remaining will diffuse to the grain boundaries. This will lead to an isolated thin layer around the crystallites. There is a stress in the crystal when Gd ions get incorporated into the lattice. Those Gd ions which accumulated at the grain boundary act as a kinetic barrier for further grain displacement and thus hinder the grain growth [18]. Decrease in grain size with increasing Gd concentration in $\left(\mathrm{Bi}_{1-\chi} \mathrm{Gd}_{x}\right)_{0.5} \mathrm{Na}_{0.5} \mathrm{TiO}_{3}(\mathrm{BNGT})$ was reported by Vijayeta Pal et al. [19].

\subsection{Energy Dispersive X-Ray Analysis}

The Energy Dispersive X-ray analysis show peaks correspond to the element present in the sample. The higher a peak in a spectrum, the more concentrated the element is in the spectrum. The EDX image shown in Figure 5 depicts the presence of Gd and $\mathrm{Zn}$ atom in the prepared samples. Absence of other elements in the spectra confirms the purity of the samples.

\subsection{Dielectric Studies}

The variation of the dielectric constant with log frequency at room temperatures is shown in Figure 6. The dielectric constant of all the samples found decreases with increasing frequency. This can be explained on the basis of Maxwell-Wagner model which is a result of the inhomogeneous medium of two-layer dielectric structure. In this model, dielectric structure is composed of well conducting grains, which are separated by the poorly conducting grain boundaries [20]. By hopping, electrons can accumulate at grain boundaries due to high resistance and produce polarization. As the frequency of the external electric field increases the hopping frequency of electrons cannot follow the alternating field. This decreases the probability of electron reaching the grain boundary and as a result polarization decreases. The results attained in this work and the explanation given above is in good agreement with the Koops phenomenological theory [21].

The observed higher value of dielectric constant at lower frequency is due to space charge polarization. While at higher frequency, polarization will lags behind the applied and hence decreases the value of dielectric constant.

Similar to dielectric constant, dielectric loss also decreases with increase in frequency and becomes constant at higher frequencies. Dielectric loss arises when the polarization lags behind the applied field and is caused by grain boundaries, impurities and imperfection in the crystal lattice [22]. Figure 7 shows the variation of dielectric loss factor with frequency. When the frequency of the applied AC electric field is smaller than the hopping frequency of electrons between $\mathrm{Zn}^{2+}$ and $\mathrm{Gd}^{3+}$ ions, the electrons follow the field and hence the loss is maximum. At higher frequencies of the applied electric field, the hopping frequency of the electron exchange between these ions cannot follow the applied field beyond certain critical frequency and the loss is minimum. The value of $\tan \delta$ is $<0.2$ in the higher frequency range showing that the material is less lossy.

To find the effect of Gd substitution on the dielectric constant of the present samples, re-plot dielectric con- 
stant of $\mathrm{Zn}_{1-\chi} \mathrm{Gd}_{x} \mathrm{O}$ as a function of Gd concentration is shown in Figure 8. It is found that dielectric constant increases slightly with small amount of Gd substitution $(x=0.2)$. Further increase in Gd concentration $(x=0.4)$ reduces the dielectric constant. Another maximum value of dielectric constant attained at $x=0.6$ and additional Gd doping $(x=0.8)$ leads to another fall in the dielectric constant. The incorporation of small amounts of $\mathrm{Gd}^{3+}$ for $\mathrm{Zn}^{2+}$ would stabilize the wurtzite structure of $\mathrm{ZnO}$ and hence reduce the number of oxygen vacancies [23]. This leads to an increase in dielectric constant. Further increase of Gd content would result a unit cell contraction which is already discussed in the XRD section. Thus, the free volume available for the displacement of ions becomes smaller and this leads to a decrease in dielectric polarization. As Gd concentration increases from $x=$ 0.4 to $x=0.6$, the dielectric constant increases again due to the presence of more grain boundaries. A saturated level of Gd content is approaching when $x$ is increased from 0.6 to 0.8. At this stage, the sample might be a composite of $\mathrm{Gd}_{2} \mathrm{O}_{3}$ and $\mathrm{ZnO}$, with a lesser dielectric constant.

The effect of Gd substitution on the dielectric loss angle is shown in Figure 9. Compared to pure ZnO, mini-

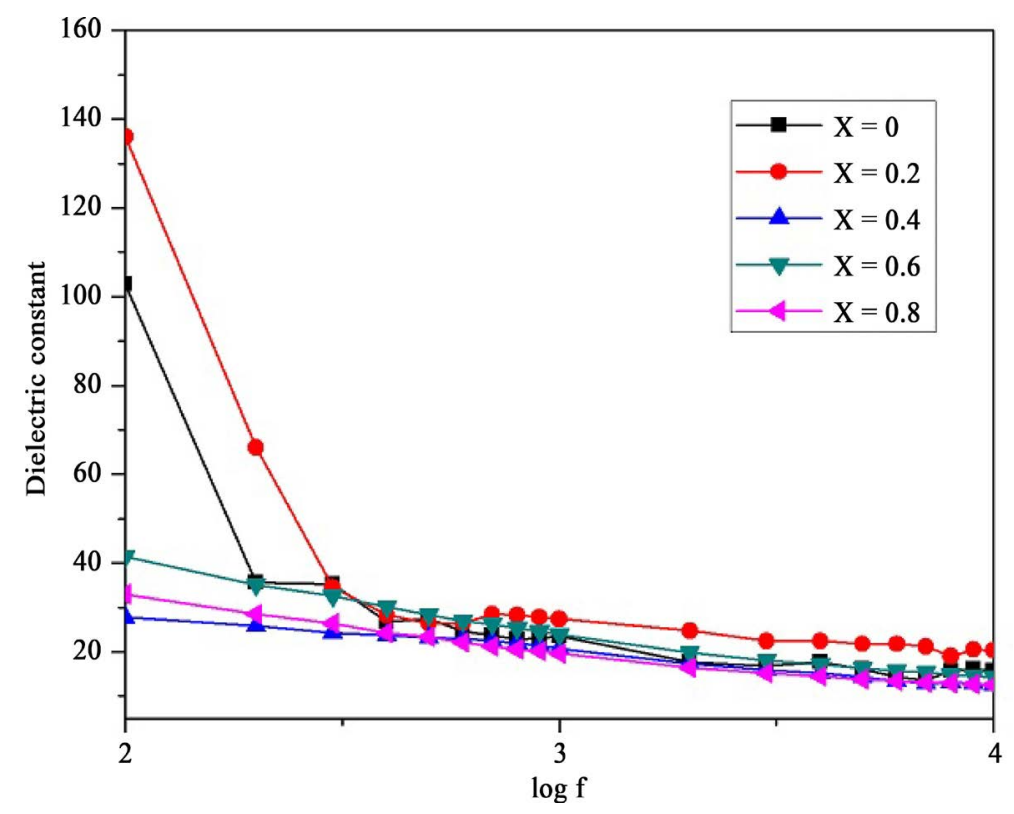

Figure 6. Variation of dielectric constant with frequency.

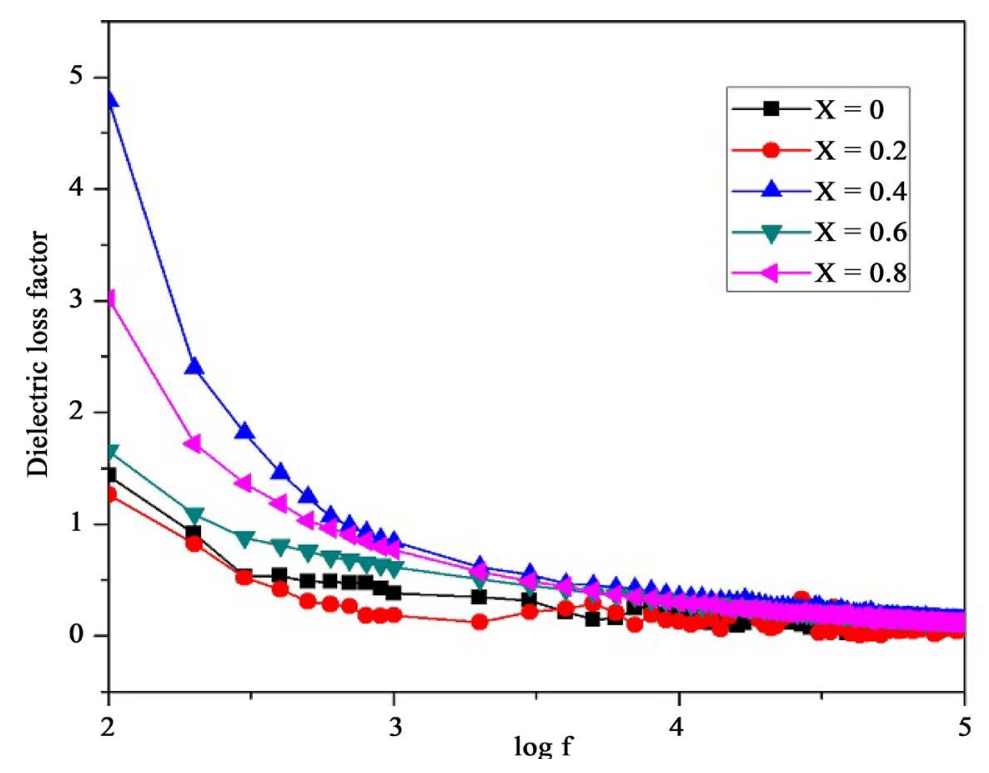

Figure 7. Variation of dielectric loss factor with frequency. 


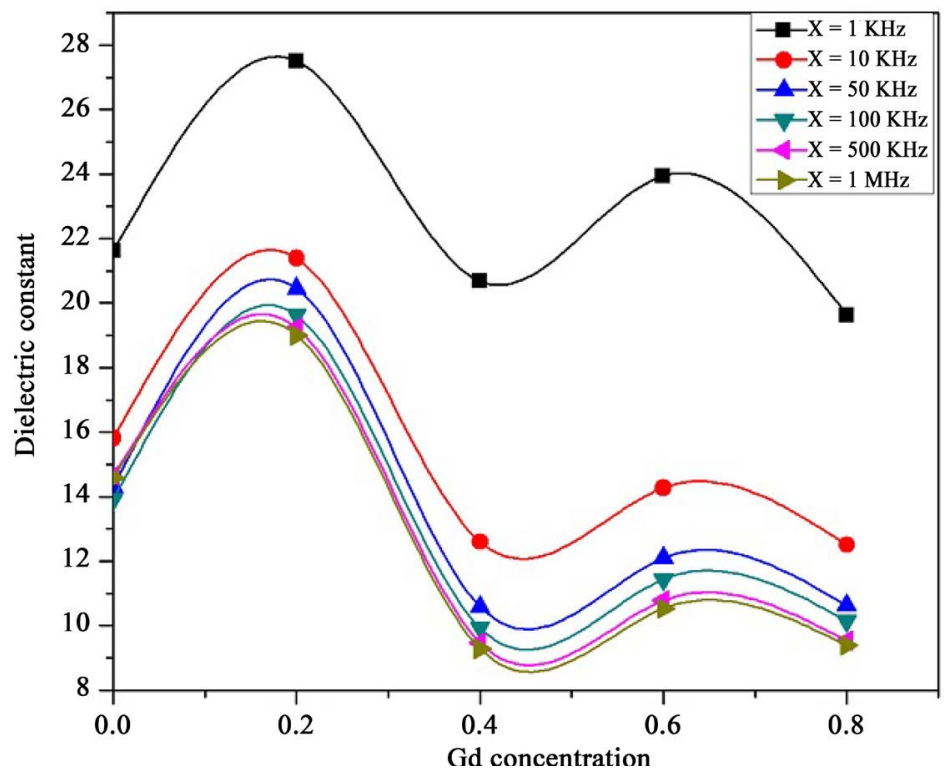

Figure 8. Variation of dielectric constant with Gd concentration for $\mathrm{Zn}_{1-x} \mathrm{Gd}_{x} \mathrm{O}$.

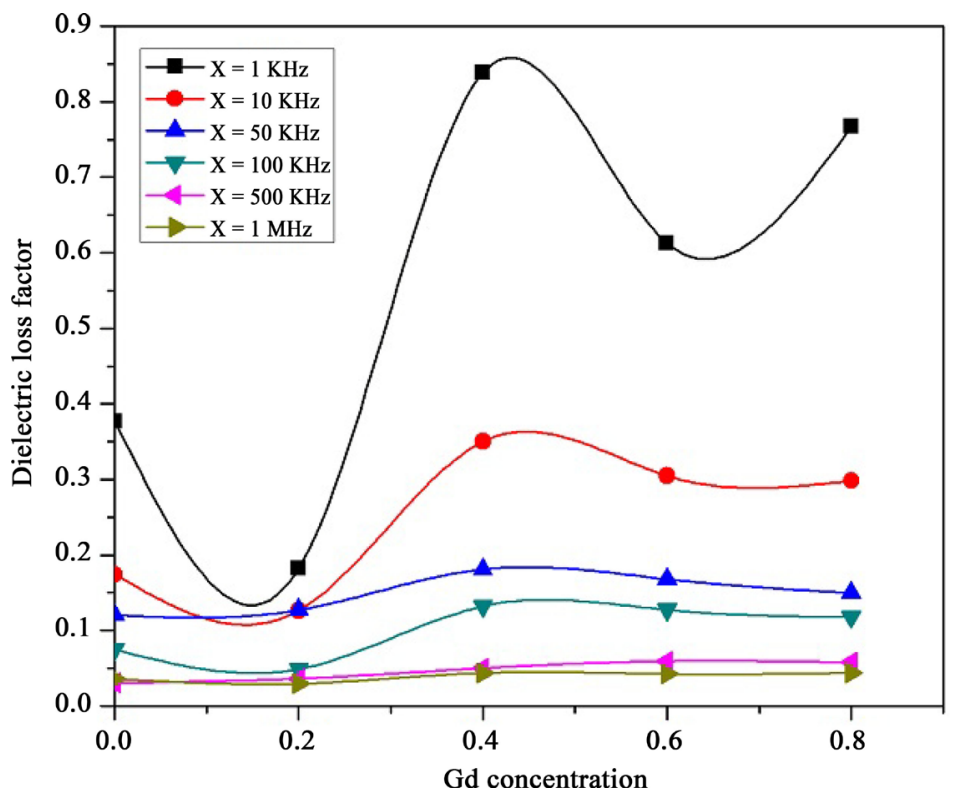

Figure 9. Variation of dielectric loss factor with Gd concentration for $\mathrm{Zn}_{1-x} \mathrm{Gd}_{x} \mathrm{O}$.

mum dielectric loss is obtained at Gd content $x=0.2$. It is also observed that all the samples have a less dielectric loss at higher frequency.

\subsection{AC Conductivity}

The conduction mechanism in the present samples was determined from the AC conductivity measurement. The variation of $\mathrm{AC}$ electrical conductivity $\left(\sigma_{\mathrm{ac}}\right)$ with frequency at room temperature is shown in Figure 10. At lower frequency, poorly conducting boundaries become more active. As a result hopping frequency between charge carriers decreases. This in turn decreases the conductivity value of the material. As frequency increases, the hopping frequency results in the increase of ac conductivity value of all the samples.

Figure 7 and Figure 10 reveal a close relationship between $\tan \delta$ and conductivity. The increase of conductivity is accompanied by an increase of the eddy current which in turn increases the energy loss $\tan \delta$ [24]. 


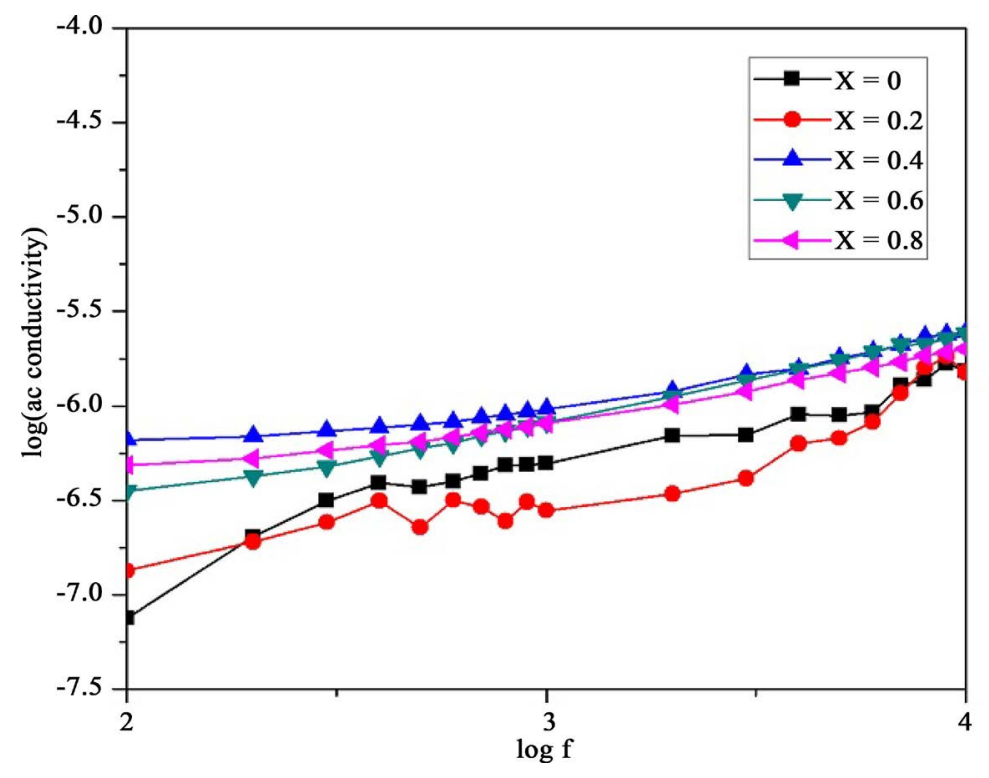

Figure 10. Variation of AC electrical conductivity with frequency.

\section{Conclusion}

Gadolinium (Gd) doped Zinc oxide ( $\mathrm{ZnO})$ nanocrystals were synthesized by the solid state reaction route by varying Gd concentration from 0 wt.\% to $0.8 \mathrm{wt} . \%$. Gd incorporation in the host lattice makes a structural distortion in $\mathrm{ZnO}$ due to the larger ionic radius of $\mathrm{Gd}$ compared to that of $\mathrm{Zn}$ and is evident from the structural studies. Increase the concentration of Gd hinders the growth of $\mathrm{Zn}_{1-x} \mathrm{Gd}_{x} \mathrm{O}$ nanocrystals and multiphase growth is observed at higher concentrations. The frequency dependence of dielectric studies revealed that for all the samples studied, the dielectric constant and dielectric loss was found decreased with increase of frequency (between 100 $\mathrm{Hz}$ and $4.5 \mathrm{MHz}$ ), whereas AC conductivity was found increased. The gadolinium doping has an important effect on the dielectric properties of $\mathrm{ZnO}$. At low $\mathrm{Gd}^{3+}$ concentrations, slightly higher value of dielectric constant is observed. The low dielectric loss at higher frequency makes this Gd doped ZnO nanocrystal as a candidate for high frequency applications.

\section{Acknowledgements}

Author (P. P. Pradyumnan) is thankful to SERB Govt. of India major research funding, DST-FIST Govt. of India, for projects sanctioned to Dept. of Physics, University of Calicut for the equipment facilities. One of the authors (Divya) acknowledges UGC-SAP for financial support.

\section{References}

[1] Zhang, Q.F., Dandeneau, C.S., Zhou, X.Y. and Cao, G.Z. (2009) ZnO Nanostructures for Dye Sensitized Solar Cells. Advanced Materials, 21, 4087-4108. http://dx.doi.org/10.1002/adma.200803827

[2] Wang, Z.L. (2004) Zinc Oxide Nanostructures: Growth, Properties and Applications. Journal of Physics: Condensed Matter, 16, R829-R858.

[3] Gurav, K.V., Fulari, V.J., Patil, U.M., Lokhande, C.D. and Joo, O. (2010) Room Temperature Soft Chemical Route for Nanofibrous Wurtzite ZnO Thin Film Synthesis. Applied Surface Science, 256, 2680-2685. http://dx.doi.org/10.1016/j.apsusc.2009.09.080

[4] Kenyon, A.J. (2002) Recent Developments in Rare-Earth Doped Materials for Optoelectronics. Progress in Quantum Electronics, 26, 225-284. http://dx.doi.org/10.1016/S0079-6727(02)00014-9

[5] Jayanthi, K., Manorama, S.V. and Chawla, S. (2013) Observation of $\mathrm{Nd}^{3+}$ Visible Line Emission in ZnO: $\mathrm{Nd}^{3+} \mathrm{Prepared}^{3}$ by a Controlled Reaction in the Solid State. Journal of Physics D: Applied Physics, 46, 325101.

[6] Achamma, G., Sharma, S.K., Chawla, S., Malik, M.M. and Qureshi, M.S. (2011) Detailed of X-Ray Diffraction and Photoluminescence Studies of Ce Doped ZnO Nanocrystals. Journal of Alloys and Compounds, 509, 5942-5946. http://dx.doi.org/10.1016/j.jallcom.2011.03.017 
[7] John, R. and Rajakumari, R. (2012) Synthesis and Characterization of Rare Earth Ion Doped Nano ZnO. Nano-Micro Letters, 4, 65-72. http://dx.doi.org/10.1007/BF03353694

[8] Sinha, N., Ray, G., Bhandari, S., Godara, S. and Kumar, B. (2014) Synthesis and Enhanced Properties of Cerium Doped ZnO Nanorods. Ceramics International, 40, 12337-12342. http://dx.doi.org/10.1016/j.ceramint.2014.04.079

[9] Divya, N.K., Aparna, P.U. and Pradyumnan, P.P. (2015) Dielectric Properties on $\mathrm{Er}^{3+}$ Doped ZnO Nanocrystals. Advances in Materials Physics and Chemistry, 5, 287-294. http://dx.doi.org/10.4236/ampc.2015.58028

[10] Divya, N.K. and Pradyumnan, P.P. (2016) Solid State Synthesis of Erbium Doped ZnO with Excellent Photocatalytic Activity and Enhanced Visible Light Emission. Materials Science in Semiconductor Processing, 41, 428-435. http://dx.doi.org/10.1016/j.mssp.2015.10.004

[11] Murmu, P.P., Kennedy, J., Ruck, B.J., Markwitz, A., Williams, G.V.M. and Rubanov, S. (2012) Structural and Magnetic Properties of Low Energy Gd Implanted ZnO Single Crystals. Nuclear Instruments and Methods in Physics Research Section B, 272, 100-103. http://dx.doi.org/10.1016/j.nimb.2011.01.041

[12] Ma, X.Y. and Wang, Z. (2012) Optical Properties of Rare Earth Gd Doped ZnO nanocrystals. Materials Science in Semiconductor Processing, 15, 227-231. http://dx.doi.org/10.1016/j.mssp.2011.05.013

[13] Gouri, M.I., Ahmed, E., Khalid, N.R., Ahmad, M., Ramzan, M., Shakoor, A. and Niaz, N.A. (2014) Gadolinium Doped ZnO Nanocrystalline Powders and Its Photocatalytic Performance for Degradation of Methyl Blue under Sunlight. Journal of Ovonic Research, 10, 89-100.

[14] Dakhel, A.A. and El-Hilo, M. (2010) Ferromagnetic Nanocrystalline Gd Doped ZnO Powder Synthesized by Coprecipitation. Journal of Applied Physics, 107, Article ID: 123905.

[15] Lin, W., Ma, R., Shao, W. and Liu, B. (2007) Structural, Electrical and Optical Properties of Gd Doped and Undoped ZnO: Al Thin Films Prepared by RF Magnetron Sputtering. Applied Surface Science, 253, 5179-5183. http://dx.doi.org/10.1016/j.apsusc.2006.11.032

[16] Subramanian, M., Thakur, P., Tanemura, M., Hihara, T., Ganesan, V., Soga, T., et al. (2010) Intrinsic Ferromagnetism and Magnetic Anisotropy in Gd Doped ZnO Thin Films Synthesized by Pulsed Spray Pyrolysis Method. Journal of Applied Physics, 108, Article ID: 053904. http://dx.doi.org/10.1063/1.3475992

[17] Mithal, D. and Kundu, T. (2013) Synthesis and Characterization of Gd Doped ZnO Nanocrystals. Asian Journal of Chemistry, 25, 12-16.

[18] Rahman, M.T., Vargas, M. and Ramana, C.V. (2014) Structural Characteristics, Electrical Conduction and Dielectric Properties of Gadolinium Substituted Cobalt Ferrite. Journal of Alloys and Compounds, 617, 547-562. http://dx.doi.org/10.1016/j.jallcom.2014.07.182

[19] Pal, V. and Dwivedi, R.K. (2012) Effect of Rare Earth Substitution on the Structural, Microstructure and Dielectric Properties of Lead Free BNT Ceramics. Advanced Materials Research, 585, 200-204. http://dx.doi.org/10.4028/www.scientific.net/AMR.585.200

[20] Thakur, A., Mathur, P. and Singh, M. (2007) Study of Dielectric Behaviour of Mn-Zn Nano Ferrites. Journal of Physics and Chemistry of Solids, 68, 378-381. http://dx.doi.org/10.1016/j.jpcs.2006.11.028

[21] Koops, C.G. (1951) On the Dispersion of Resistivity and Dielectric Constant of Some Semiconductors at Audio Frequencies. Physical Review, 83, 121-124. http://dx.doi.org/10.1103/PhysRev.83.121

[22] Mangalaraja, R.V., Manohar, P. and Gnanam, F.D. (2004) Electrical and Magnetic Properties of $\mathrm{Ni}_{0.8} \mathrm{Zn}_{0.2} \mathrm{Fe}_{2} \mathrm{O}_{4} / \mathrm{Silica}$ Composite Prepared by Sol-Gel Method. Journal of Materials Science, 39, 2037-2042. http://dx.doi.org/10.1023/B:JMSC.0000017766.07079.80

[23] Fanggao, C., Guilin, S., Kun, F., Ping, Q. and Qijun, Z. (2006) Effect of Gadolinium Substitution on Dielectric Properties of Bismuth Ferrite. Journal of Rare Earths, 24, 273-276. http://dx.doi.org/10.1016/S1002-0721(07)60379-2

[24] Satter, A.A. and Samy, A.R. (2003) Dielectric Properties of Rare Earth Substituted Cu-Zn Ferrites. Physica Status Solidi(a), 200, 415-422. http://dx.doi.org/10.1002/pssa.200306663 